\title{
The Volume of a Differentiable Stack
}

\author{
ALAN WEINSTEIN \\ Department of Mathematics, University of California, Berkeley, CA 94720, USA. \\ e-mail: alanw@math.berkeley.edu
}

Received: 19 September 2008 / Revised: 22 August 2009 / Accepted: 23 August 2009 Published online: 22 September 2009 - (C) The Author(s) 2009. This article is published with open access at Springerlink.com

\begin{abstract}
We extend the notion of the cardinality of a discrete groupoid (equal to the Euler characteristic of the corresponding discrete orbifold) to the setting of Lie groupoids. Since this quantity is an invariant under equivalence of groupoids, we call it the volume of the associated stack rather than of the groupoid itself. Since there is no natural measure in the smooth case like the counting measure in the discrete case, we need extra data to define the volume. This data has the form of an invariant section of a natural line bundle over the base of the groupoid. Invariant sections of a square root of this line bundle constitute an "intrinsic Hilbert space" of the stack.
\end{abstract}

Mathematics Subject Classification (2000). Primary 58H05; Secondary 53D17.

Keywords. Lie groupoid, Lie algebroid, modular class, differentiable stack.

\section{Introduction}

As part of a larger program of groupoidification, the cardinality of a groupoid $G \Longrightarrow G_{0}$ with finitely many orbits and finite isotropy groups was defined by Baez and Dolan [1] to be the sum over orbits of the reciprocal of the orders of the isotropy groups. This sum is well defined because the isotropy groups of different elements of an orbit are isomorphic. It represents the total mass of the "natural measure" of such a groupoid defined by Kim [11] in connection with a Lefschetz formula for equivariant cohomology. This in turn was inspired by the same expression in Behrend's Lefschetz formula [3] for the Frobenius automorphism on algebraic stacks. The expression also appears as the Euler characteristic of a 0-dimensional orbifold.

Baez and Dolan give many examples of and reasons for their definition. For instance, if the groupoid is the transformation groupoid associated with the action of a group $H$ on a space $X$, its cardinality is the quotient $\#(X) / \#(H)$ of the number of elements $\#(X)$ in $X$ by the order $\#(H)$ of $H$. In particular, if $X$ is a single point, the cardinality is $1 / \#(H)$.

A. Weinstein's research was partially supported by NSF Grant DMS-0204100. 
Since the cardinality is clearly an invariant under equivalence of groupoids, we prefer to think of it as an invariant $\#\left(G_{0} / / G\right)$ of the quotient stack $^{1} G_{0} / / G$ (or $X / / H$ in the case of a transformation groupoid). The terminology is consistent with the usual one when the action is free, so that $X / / H$ is simply the (stack associated to the) set $X / H$, and $\#(X / H)=\#(X) / \#(H)$. On the other hand, when $X$ is a point, $X / / H$ is the universal classifying stack $B H$, so $\#(B H)$ becomes $1 / \#(H)$. As Baez and Dolan themselves note, this result is consistent with the idea that $B H=\mathrm{pt} / / H$ may be thought of as "one \# $(H)$ 'th of a point". (Do not try to read this aloud!)

The aim of the present work is to extend the notion of groupoid cardinality from the discrete to the differentiable setting, i.e. to Lie groupoids and their associated smooth stacks. We call our extended notion the volume of a stack, thinking of the cardinality as a geometric rather than a topological quantity. It is clear that we now need additional data, namely measures which generalize the counting measures on sets and groups which are implicit in the discrete situation. It turns out that the appropriate data are all contained in a $G$-invariant section of the bundle $Q_{A} \stackrel{\text { def }}{=} \bigwedge^{\text {top }} A \otimes \bigwedge^{\text {top }} T^{*} G_{0}$ defined in [10], where $A$ is the Lie algebroid of $G$. In fact, when $G=G_{0}$, such a section is just a volume element $b$ on $G_{0}$, while when $G_{0}$ is a point, the section is the value at the identity of a bi-invariant multivector field of top degree, or "inverse volume element", $a^{-1}$ (the inverse of the volume element $a$ ) on the group $G$. In the former case, our definition will give the integral $^{2}$ of $b$ over $G_{0}$, i.e. the volume of $G_{0}$ with the measure given by $b$; in the latter, our definition will give the reciprocal of the integral over $G$ of $a$. (If $a=0$, or if $G$ is noncompact, the invariant is defined to be 0 .)

We will see that, in the discrete case, our invariant reduces to that of Baez and Dolan, with the additional flexibility that we can replace the counting measures by arbitrary measures subject to an invariance condition. Furthermore, if $G$ is the groupoid associated with a smooth action of a Lie group $H$ on a manifold $X$, a special kind of invariant section of $Q_{A}$ is the product of an $H$-invariant volume element $b$ on $X$ and a bi-invariant, nowhere-vanishing volume element $a$ on $H$, and for such a section our volume for $X / / H$ will be the quotient $\int_{X} b / \int_{H} a$. This quotient makes clear sense when $X$ and $H$ are compact; when this is not the case, but the action of $H$ on $X$ is still proper and cocompact (i.e. having compact orbit space $^{3}$ ), our invariant continues to be well-defined. Note that $H$ is not required to be unimodular; when it is not, the non-invariance of a Haar measure under conjugation must be countered exactly by the non-invariance of a measure on $X$.

More generally, we will see that any invariant section of $Q_{A}$ defines a measure on the coarse moduli (or ordinary quotient, or orbit) space $G_{0} / G$ whose integral

\footnotetext{
${ }^{1}$ We refer to $[4,5]$ for background material on differentiable stacks.

${ }^{2}$ We will assume here and usually elsewhere that all our manifolds are oriented; the nonorientable case can be handled with the use of densities instead of forms.

${ }^{3}$ Perhaps another appropriate name for this condition would be "coproper".
} 
over any relatively compact open subset $U$ is the volume of the inverse image of $U$ under the natural projection from $G_{0} / / G$ to $G_{0} / G$. Another way of expressing this is to say that a section of $Q_{A}$ is a "smooth measure" on the stack, and the ordinary measure on the orbit space is its push-forward. We can also consider the line bundle associated to the frame bundle of $Q_{A}$ via the homomorphism $a \rightarrow|a|^{1 / 2}$ from the multiplicative reals to themselves. As in [20], we denote this line bundle by $\left|Q_{A}\right|^{1 / 2}$. $G$ still acts on this bundle, and its space of compactly supported invariant sections carries a natural inner product. The Hilbert space completion of this space is an "intrinsic $L^{2}$ space" for the stack $G_{0} / / G$. In a similar way, one may construct $L^{p}$ spaces in duality, and spaces of distributions.

A natural next step from here should be to attach a vector space (sections, or distributional sections of $Q_{A}$ or one of its powers) to each groupoid $G$, and to attach a linear map to each morphism in some nice category. Here, we think of the volume of a stack as a linear map $\mathbb{R} \rightarrow \mathbb{R}$ associated to the diagram of groupoids pt $\leftarrow G \rightarrow$ pt. Such a diagram is a special case of a span $G \leftarrow S \rightarrow H$, which, according to the degroupoidification program described in [2], should be considered as a kind of morphism from $G$ to $H$.

It may be that the appropriate morphisms to consider when extending from the finite case should be those which are between stacks of the same dimension, or perhaps submersions of stacks, for which one could try to integrate over the fibres. Here, an extension to groupoids of the relative modular class for Lie algebroid morphisms in [12] should come into play.

From the point of view of microlocal analysis, one might even want to consider more general geometric morphisms between stacks, encoded by lagrangian submanifolds and symbols, which would induce pseudodifferential or even Fourier integral operators between the corresponding vector spaces. A prerequisite for doing this would be a correct definition of the cotangent bundle of a stack as a "symplectic stack". A symplectic stack cannot be simply a stack defined by a symplectic groupoid, since such a stack has no symplectic structure of its own. Rather, the cotangent stack should be presented by a "groupoid in the symplectic category", in which the groupoid structure operations are canonical relations which may even be multiply-defined. In fact, one should get such an object when applying to the structure operations in a Lie groupoid the functor which assigns to each mapping (or relation) between manifolds the conormal bundle to its graph.

\section{First Definition and the Finite Case}

In this section, we give a provisional definition of the volume of the stack $G_{0} / / G$ (sometimes denoted $B G$ ) presented by a groupoid $G \Longrightarrow G_{0}$ (sometimes denoted simply as $G$ ) in terms of an invariant section $\lambda$ of $Q_{A}$, where $A$ is the Lie algebroid of $G$. We will see that this definition has two difficulties. First of all, it depends on the decomposition of $\lambda$ as the quotient of a section $b$ of $\wedge^{\text {top }} T^{*} G_{0}$ by a section $a$ of $\Lambda^{\text {top }} A^{*}$, and it is not clear that it is independent of the decomposition. 
Second, it produces a sensible result only when the source and target maps from $G$ to $G_{0}$ are proper, although only properness of the groupoid, i.e. properness of the map $(l, r): G \rightarrow G_{0} \times G_{0}$, should be needed. (Here and later, the letters $l$ and $r$ will denote the source and target maps from $G$ to $G_{0}$. The reader may choose which is to be the source and which is the target, but we will insist that the product $g h$ is defined when $r(g)=l(h)$.)

In the next section, we will see how to remove the difficulties with our definition. But before even giving the definition, we will look more closely at the finite case.

DEFINITION 2.1. [1] If $G=G \Longrightarrow G_{0}$ is a groupoid with finite isotropy groups and finitely many orbits, we define the cardinality of the stack $G_{0} / / G$ to be the sum

$$
\#\left(G_{0} / / G\right) \stackrel{\text { def }}{=} \sum_{\mathcal{O}} \#\left(G_{\mathcal{O}}\right)^{-1},
$$

where $\mathcal{O}$ ranges over the orbit space $G_{0} / G$, and $\#\left(G_{\mathcal{O}}\right)$ denotes the order of the isotropy group of any element of $\mathcal{O}$. (The cardinality of the empty groupoid is zero.)

It is clear from the definition that equivalent groupoids have the same cardinality, so that this quantity is really an invariant of the stack, independent of its presentation by a groupoid.

As Baez and Dolan [1] note, the corresponding sum may be convergent for some interesting groupoids with infinitely many orbits. For example, the cardinality of the stack presented by the groupoid of all finite sets and their bijections (or an equivalent groupoid whose objects form a set rather than a class) is $e=2.718 \ldots$.

It is not so simple to transfer Definition 2.1 to the smooth case, since the orbit space is generally not a smooth manifold. Instead, we will reformulate the definition on the basis of the following simple fact, which is a special case of Theorem 2.4 .

PROPOSITION 2.2. For any finite groupoid $G \Longrightarrow G_{0}$,

$$
\#\left(G_{0} / / G\right)=\sum_{y \in G_{0}} \#\left(r^{-1}(y)\right)^{-1} .
$$

Proposition 2.2 suggests defining the volume of $G_{0} / / G$, when $G$ is a Lie groupoid, by integrating over $G_{0}$ the reciprocals of the volumes of the fibres of the surjective submersion $r: G \rightarrow G_{0}$. To do this, we need a measure on $G_{0}$ and measures along the fibres of $r$. Using this data, we make the following provisional definition, assuming as usual that $G$ and $G_{0}$ are oriented.

DEFINITION 2.3. Let $G \Longrightarrow G_{0}$ be a compact Lie groupoid (i.e. $G$ is a compact manifold, so that $G_{0}$ and the fibres of $r$ are also compact) with Lie algebroid $A$. 
Let $a$ be a nowhere vanishing section of $\bigwedge^{\text {top }} A^{*}$ and $b$ a section of $\bigwedge^{\text {top }} T^{*} G_{0}$. The volume of the stack $G_{0} / / G$ with data $(a, b)$ is defined as

$$
\operatorname{vol}_{(a, b)}\left(G_{0} / / G\right) \stackrel{\text { def }}{=} \int_{y \in G_{0}}\left(\int_{r^{-1}(y)} a_{r}\right)^{-1} b .
$$

In this definition, $a_{r}$ is the right-invariant form, defined along the tangent bundle to the $r$-fibres, whose values along the unit section are given by $a$. (The Lie algebroid $A$ is identified with the tangent bundle along the units to the $r$-fibres.)

To show that this definition depends only on the stack and not on the presenting groupoid, we cannot even begin without having a way of moving the data $(a, b)$ from one groupoid to any equivalent one. But this is not possible; what is transferable between equivalent groupoids is only the product $a^{-1} b$, which is a section of the tensor product line bundle $Q_{A} \stackrel{\text { def }}{=} \bigwedge^{\text {top }} A \otimes \bigwedge^{\text {top }} T^{*} G_{0}$. (See [12] and Section 4.) We thus need to show that $\operatorname{vol}_{(a, b)} G_{0} / / G$ depends only the product $a^{-1} b$ and not on the individual factors; i.e. that the volume is unchanged when $a$ and $b$ are multiplied by the same nonvanishing function $\theta$ on $G_{0}$.

To see how to proceed, we return to the finite case, where the bundles $A$ and $T G_{0}$ have 0 -dimensional fibres, so sections of their top exterior powers (and of the top exterior powers of their duals) are simply scalar functions. With data $(a, b)$, Definition 2.3 in the case of a finite groupoid becomes

$$
\operatorname{vol}_{(a, b)}\left(G_{0} / / G\right) \stackrel{\text { def }}{=} \sum_{y \in G_{0}}\left(\sum_{g \in r^{-1}(y)} a(l(g))\right)^{-1} b(y),
$$

which becomes the cardinality formula in Proposition 2.2 when $a$ and $b$ are unity.

For the outer sum, we may sum over each orbit and then sum over the orbit space, i.e.

$$
\operatorname{vol}_{(a, b)}\left(G_{0} / / G\right)=\sum_{\mathcal{O} \in G_{0} / G} \sum_{y \in \mathcal{O}}\left(\sum_{g \in r^{-1}(y)} a(l(g))\right)^{-1} b(y) .
$$

For each orbit $\mathcal{O}$, we have

$$
S_{\mathcal{O}} \stackrel{\text { def }}{=} \sum_{y \in \mathcal{O}}\left(\sum_{g \in r^{-1}(y)} a(l(g))\right)^{-1} b(y)=\sum_{y \in \mathcal{O}}\left(\sum_{x \in \mathcal{O}} \sum_{g \in l^{-1}(x) \cap r^{-1}(y)} a(x)\right)^{-1} b(y) .
$$

Now the number of elements in $l^{-1}(x) \cap r^{-1}(y)$ depends only on the orbit $\mathcal{O}$ and is equal to the cardinality $\#\left(G_{\mathcal{O}}\right)$ of the typical isotropy group. Hence, we have

$$
S_{\mathcal{O}}=\#\left(G_{\mathcal{O}}\right)^{-1}\left(\sum_{x \in \mathcal{O}} a(x)\right)^{-1} \sum_{y \in \mathcal{O}} b(y)
$$


In general, $S_{\mathcal{O}}$ will depend on all the values of $b / a$ on $\mathcal{O}$, but if $\lambda=b / a$ is constant on orbits (i.e. a $G$-invariant section of the trivial bundle $Q_{A}$ ), it only depends on the constant value $\lambda(\mathcal{O})$ of that section on the orbit, and we obtain the final formula:

$$
\operatorname{vol}_{\lambda}\left(G_{0} / / G\right)=\sum_{\mathcal{O} \in G_{0} / G} \#\left(G_{\mathcal{O}}\right)^{-1} \lambda(\mathcal{O}) .
$$

Since the right hand side is clearly invariant under equivalence of groupoids, we have the following result.

THEOREM 2.4. Let $G \Longrightarrow G_{0}$ be a finite groupoid. Let $a$ and $b$ be functions on $G_{0}$ such that $a$ is nowhere vanishing and the quotient $\lambda=b / a$ is $G$-invariant, so that $\lambda$ may be considered as a function on $G_{0} / G$, or a $G$-invariant section of $Q_{A}$, where $A$ is the (zero-dimensional) Lie algebroid of $G$.

Then the quantity

$$
\sum_{y \in G_{0}}\left(\sum_{g \in r^{-1}(y)} a(l(g))\right)^{-1} b(y)
$$

is equal to

$$
\sum_{\mathcal{O} \in G_{0} / G} \#\left(G_{\mathcal{O}}\right)^{-1} \lambda(\mathcal{O}) .
$$

In particular, it depends on $a$ and $b$ only via their quotient $\lambda$.

Furthermore, given an equivalence between $G$ and another finite groupoid $G^{\prime}$, with Lie algebroid $A^{\prime}$, there is a bijective correspondence between $G$-invariant sections of $Q_{A}$ and $Q_{A^{\prime}}$, and if $\lambda^{\prime}=b^{\prime} / a^{\prime}$ is the section corresponding to $\lambda=b / a$, then

$$
\sum_{y \in G_{0}}\left(\sum_{g \in r^{-1}(y)} a(l(g))\right)^{-1} b(y)=\sum_{y^{\prime} \in G_{0}^{\prime}}\left(\sum_{g^{\prime} \in r^{\prime-1}\left(y^{\prime}\right)} a^{\prime}\left(l^{\prime}\left(g^{\prime}\right)\right)\right)^{-1} b^{\prime}\left(y^{\prime}\right) .
$$

We may therefore make the following definition.

DEFINITION 2.5. Let $G \Longrightarrow G_{0}$ be a groupoid with finite isotropy groups, $G_{0} / / G$ the corresponding stack, and $\pi: G_{0} / / G \rightarrow G_{0} / G$ the natural projection. Then any function $\lambda$ on $G_{0} / G$ (i.e. $G$-invariant section of $Q_{A}$, where $A$ is the Lie algebroid of $G$ ) defines a measure $\mu_{\lambda}$ on $G_{0} / / G$ for which the "measurable sets" are the preimages under $\pi$ of finite subsets of $G_{0} / G$, and the measure of such a subset $\mathcal{U}$ is defined by

$$
\mu_{\lambda}\left(\pi^{-1}(\mathcal{U})\right) \stackrel{\text { def }}{=} \sum_{\mathcal{O} \in \mathcal{U}} \#\left(G_{\mathcal{O}}\right)^{-1} \lambda(\mathcal{O})
$$

We may therefore consider $\mu_{\lambda}$ as a volume element, or measure, on $G_{0} / / G$; its push-forward under the natural projection $\pi: G_{0} / / G \rightarrow G_{0} / G$ is the measure which assigns to each point $\mathcal{O}$ the measure $\#\left(G_{\mathcal{O}}\right)^{-1} \lambda(\mathcal{O})$. 


\section{The Differentiable Case}

Definition 2.3 has the virtue that it clearly leads to a finite result for any compact groupoid, but its invariance properties are hard to verify directly. We would like to imitate the orbit decomposition method of the previous section, but this works nicely only when the groupoid is strongly regular in the sense that the decomposition into orbits is a fibration. Our strategy will be to apply the orbit decomposition on the strongly regular part of the groupoid, whose complement turns out to be negligible as far as integration is concerned. The latter fact follows immediately from the slice theorem in [19] and Zung's linearization theorem [21] for proper groupoids, since the orbit structure of a proper groupoid is locally like that of the action of a compact group, for which there is a principal orbit type.

Assume now, then, that $G \Longrightarrow G_{0}$ is a strongly regular, compact (hence proper) groupoid, and let $f: G_{0} \rightarrow G_{0} / G$ be the natural projection. Then the integral over $G_{0}$ in Definition 2.3 of the function

$$
\left(\int_{r^{-1}(y)} a_{r}\right)^{-1}
$$

times the volume element $b$ can be written as iterated integral-first over the fibres of $f$, i.e. over the individual orbits, and then over the orbit space. To do this, we will need to decompose $b$ as the product of a volume element along the $G$-orbits and one on the orbit space.

The inner integral in Definition 2.3 can also be written as an iterated integral. In fact, the restriction to $r^{-1}(y)$ of the map $l$ is a principal fibration over the orbit $G y$ with structure group the isotropy group $G_{y}$ acting from the right by groupoid multiplication. Again, to get an iterated integral, we must decompose the integrand $a_{r}$ as a product of a volume element along the $G_{y}$-orbits and one along the $G$-orbit Gy.

To obtain the decompositions above we must make a choice. To see what to do, we recall the exact sequence of vector bundles over $G_{0}$,

$$
0 \rightarrow \operatorname{ker} \rho \rightarrow A \rightarrow T G_{0} \rightarrow \operatorname{coker} \rho \rightarrow 0,
$$

where $\rho: A \rightarrow T G_{0}$ is the anchor map of $G$, which may be identified with the restriction of $T l: T G \rightarrow T G_{0}$ to the kernel of $T r$ along the unit section. The kernel of $\rho$ is thus the bundle of Lie algebras of the isotropy groups, while its cokernel is the conormal bundle to the foliation by $G$-orbits. The standard "alternating product" rule for top exterior powers in an exact sequence yields a natural isomorphism

$$
Q_{A}=\bigwedge_{\text {top }}^{\text {top }} A \otimes \bigwedge^{*} T^{*} \approx \bigwedge_{0}^{\text {top }} \operatorname{ker} \rho \otimes \bigwedge^{\text {top }}(\operatorname{coker} \rho)^{*} .
$$


More explicitly, the isomorphism in (1) comes from the natural isomorphisms $A / \operatorname{ker} \rho \approx \rho(A)$ and $\operatorname{coker} \rho \approx T G_{0} / \rho(A)$.

Now, given data $a$ and $b$ as above for which $\lambda=a^{-1} b$ is a $G$-invariant section of $Q_{A}$, there is a corresponding $G$-invariant section, which we also denote by $\lambda$, of $\bigwedge^{\text {top }} \operatorname{ker} \rho \otimes \bigwedge^{\text {top }}(\operatorname{coker} \rho)^{*}$. Since $G$ is a proper groupoid acting on the line bundle $\Lambda^{\text {top }} \operatorname{ker} \rho$ (which we assume, as usual, to be orientable), we can find a nonvanishing invariant section ${ }^{4} \alpha$ of this bundle. We may then write $\lambda$ as $\alpha^{-1} \beta$, and the invariance of $\lambda$ implies that $\beta$ is an invariant section of $\wedge^{\text {top }}(\operatorname{coker} \rho)^{*}$.

Remark 3.1. Note that we could not have imposed the invariance requirement on $a$ and $b$, since the line bundles of which they are sections do not have natural actions of $G$, only actions up to homotopy. On the other hand, it is only in the regular case that we can speak of smooth sections of the kernel and cokernel of $\rho$.

The section $\beta$ gives a bi-invariant volume element on each isotropy group of $G$ which is "the same" on all the isotropy groups over a given orbit. We thus have a well-defined volume function $\operatorname{vol}_{\beta}\left(G_{\mathcal{O}}\right)$ on the orbit space $G_{0} / G$. On the other hand, $\alpha$ is a $G$-invariant volume element on the orbit space. We therefore have the well defined expression

$$
\int_{\mathcal{O} \in G_{0} / G} \operatorname{vol}_{\beta}\left(G_{\mathcal{O}}\right)^{-1} \alpha,
$$

which reduces to

$$
\sum_{\mathcal{O} \in G_{0} / G} \#\left(G_{\mathcal{O}}\right)^{-1} \lambda(\mathcal{O})
$$

in the finite case. Furthermore, it is clear that this expression does not depend on the choice of $\beta$, since multiplying it by a function $\theta$, which must be invariant, requires multiplication of $\alpha$ by the same function, and the effects of the two multiplications cancel one another.

We will now prove the following extension of Theorem 2.4, which validates Definition 2.3

THEOREM 3.2. Let $G \Longrightarrow G_{0}$ be a compact Lie groupoid with Lie algebroid A for which the map $r: G \rightarrow G_{0}$ is proper (hence a locally trivial fibration). Let $a$ and $b$ be sections of $\bigwedge^{\text {top }} A^{*}$ and $\bigwedge^{\text {top }} T^{*} G_{0}$, respectively, such that a is nowhere vanishing, and the quotient $\lambda=a^{-1} b$ is a G-invariant section of $Q_{A} \stackrel{\text { def }}{=} \bigwedge^{\text {top }} A \otimes \bigwedge^{\text {top }} T^{*} G_{0}$.

Then the quantity

$$
\int_{y \in G_{0}}\left(\int_{r^{-1}(y)} a_{r}\right)^{-1} b
$$

${ }^{4}$ To construct such a section, start with any non-vanishing section, then average over $G$, using a cutoff function [16] as in the proof of the vanishing theorem for groupoid cohomology in [6]. 
is equal to

$$
\int_{\mathcal{O} \in G_{0} / G} \operatorname{vol}_{\beta}\left(G_{\mathcal{O}}\right)^{-1} \alpha,
$$

where $\alpha$ and $\beta$ are any G-invariant sections of $\bigwedge^{\text {top }}(\operatorname{ker} \rho)^{*}$ and $\bigwedge^{\text {top }}(\operatorname{coker} \rho)^{*}$, respectively, such that $\alpha$ is nowhere vanishing and $\alpha^{-1} \beta$ corresponds to $\lambda$ under the natural isomorphism (1). In particular, it depends on $a$ and $b$ only through their quotient $\lambda$.

Furthermore, given an equivalence between $G$ and another compact groupoid $G^{\prime}$, with Lie algebroid $A^{\prime}$, there is a bijective correspondence between $G$-invariant sections of $Q_{A}$ and $Q_{A^{\prime}}$, and if $\lambda^{\prime}=b^{\prime} / a^{\prime}$ is the section corresponding to $\lambda=b / a$, then

$$
\int_{y \in G_{0}}\left(\int_{r^{-1}(y)} a_{r}\right)^{-1} b=\int_{y^{\prime} \in G_{0}^{\prime}}\left(\int_{r^{\prime-1}\left(y^{\prime}\right)} a_{l^{\prime}}^{\prime}\right)^{-1} b^{\prime} .
$$

Proof. Since a compact Lie groupoid is proper, and a proper groupoid is locally equivalent to action groupoids for actions of compact groups, the natural projection $\pi: G_{0} \rightarrow G_{0} / G$ is a fibration with compact fibres when restricted to an invariant open subset $\mathcal{V} \subset G_{0}$ whose complement has positive codimension and therefore does not contribute to the integral in the theorem. We may therefore take the integral over this strongly regular set and will use the Fubini theorem, imitating the summation proof of Theorem 2.4. We denote by $\omega$ the unique section of $\bigwedge^{\text {top }} \rho(A)^{*}$ for which $b=\omega \beta$ (from which it follows that $a=\alpha \omega$ ).

$$
\begin{aligned}
\int_{y \in G_{0}}\left(\int_{g \in r^{-1}(y)} a_{r}\right)^{-1} b & =\int_{y \in \mathcal{V}}\left(\int_{r^{-1}(y)} a_{r}\right)^{-1} b= \\
& =\int_{y \in \mathcal{V}}\left(\int_{r^{-1}(y)} a_{r}\right)^{-1} \omega \beta= \\
& \left.=\int_{\mathcal{O} \in \mathcal{N}(\mathcal{V})}\left[\underset{y \in \mathcal{O}}{\int} \underset{r^{-1}(y)}{\int(\alpha \omega)_{l}}\right)^{-1} \omega\right]
\end{aligned}
$$

But, using the fibration $l: r^{-1}(y) \rightarrow \mathcal{O}$, we have

$$
\int_{r^{-1}(y)}(\alpha \omega)_{l}=\int_{x \in \mathcal{O}}\left(\int_{l^{-1}(x) \cap r^{-1}(y)} \alpha\right) \omega=
$$




$$
\begin{aligned}
& =\int_{x \in \mathcal{O}} \operatorname{vol}_{\alpha}\left(G_{\mathcal{O}}\right) \omega= \\
& =\operatorname{vol}_{\alpha}\left(G_{\mathcal{O}}\right) \int_{\mathcal{O}} \omega .
\end{aligned}
$$

Combining the last two calculations, we obtain:

$$
\begin{aligned}
\int_{y \in G_{0}}\left(\int_{g \in r^{-1}(y)} a_{r}\right)^{-1} & =\int_{\mathcal{O} \in \mathcal{\pi}(\mathcal{V})}\left[\int_{y \in \mathcal{O}}\left(\operatorname{vol}_{\alpha}\left(G_{\mathcal{O}}\right) \int_{\mathcal{O}} \omega\right)^{-1} \omega\right] \beta= \\
& =\int_{\mathcal{O} \in \mathcal{\pi}(\mathcal{V})} \operatorname{vol}_{\alpha}\left(G_{\mathcal{O}}\right)^{-1}\left(\int_{\mathcal{O}} \omega\right)^{-1}\left(\int_{\mathcal{O}} \omega\right) \beta= \\
& =\int_{\mathcal{O} \in \pi(\mathcal{V})} \operatorname{vol}_{\alpha}\left(G_{\mathcal{O}}\right)^{-1} \beta= \\
& =\int_{G_{0} / G} \operatorname{vol}_{\alpha}\left(G_{\mathcal{O}}\right)^{-1} \beta .
\end{aligned}
$$

(For the last equality, we simply take integration over the strongly regular part as a definition of integration over the singular space $G_{0} / G$. Convergence of the integral over the noncompact complement of the singular points is guaranteed by its equality with the expression involving nonsingular integrals over $G$ and the $r$-fibres.)

We may therefore make the following definition.

DEFINITION 3.3. Let $G \Longrightarrow G_{0}$ be a proper Lie groupoid, $G_{0} / / G$ the corresponding stack, $\pi: G_{0} / / G \rightarrow G_{0} / G$ the natural projection, and $\mathcal{V} \subseteq G_{0}$ the strongly regular set. Then any $G$-invariant section $\lambda$ of $Q_{A}$, where $A$ is the Lie algebroid of $G$, defines a (signed) measure $\mu_{\lambda}$ on $G_{0} / / G$ for which the "measurable sets" are the preimages under $\pi$ of relatively compact open subsets of $G_{0} / G$, and the measure of such sets is defined as

$$
\mu_{\lambda}\left(\pi^{-1}(\mathcal{U})\right) \stackrel{\text { def }}{=} \int_{\mathcal{O} \in \mathcal{U} \cap \pi(\mathcal{V})} \operatorname{vol}_{\beta}\left(G_{\mathcal{O}}\right)^{-1} \alpha,
$$

where $\alpha$ and $\beta$ are as in Theorem 3.2.

We may therefore consider $\lambda$ as a volume element, or measure on the stack $G_{0} / / G$. Its push-forward under the natural projection $\pi: G_{0} / / G \rightarrow G_{0} / G$ is the measure which assigns to each relatively compact open subset $\mathcal{U}$ of $G_{0} / G$ the integral of the smooth form $\operatorname{vol}_{\beta}\left(G_{\mathcal{O}}\right)^{-1} \alpha$ over the intersection of $\mathcal{U}$ with the part of $G_{0} / G$ over which $G$ is strongly regular. 


\section{Morita Invariance}

Although the formula in Definition 3.3 shows that the volume of a stack clearly depends only on Morita invariant data for a groupoid, it still needs to be shown that any Morita equivalence between groupoids $G^{\prime}$ and $G^{\prime \prime}$ with Lie algebroids $A^{\prime}$ and $A^{\prime \prime}$ induces a natural isomorphism between the spaces of smooth invariant sections of the line bundles $Q_{A^{\prime}}$ and $Q_{A^{\prime \prime}}$. These spaces may therefore be considered as representatives of "the space of volume elements of a stack".

The argument for invariance begins with the special case of the inclusion of an open subset of $G_{0}$ which is full, i.e. which intersects every $G$-orbit.

LEMMA 4.1. Let $G \Longrightarrow G_{0}$ be a groupoid, $G_{0}^{\prime} \subseteq G_{0}$ a full open subset, $G^{\prime} \Longrightarrow G_{0}^{\prime}$ the restriction of $G$ to $G_{0}^{\prime}$. Then the restriction operation defines an isomorphism from the smooth $G$-invariant sections of $Q_{A}$ to the smooth $G^{\prime}$-invariant sections of $Q_{A^{\prime}}$.

Proof. Since $G_{0}^{\prime}$ intersects each orbit, each invariant section $\lambda^{\prime}$ of $Q_{A^{\prime}}$ extends uniquely to an invariant section of $Q_{A}$. It is clear that restriction preserves smoothness; we just have to show the same for extension, i.e. that an invariant section of $Q_{A}$ is smooth if and only if its restriction to some full open subset is smooth.

It is easy to prove the last statement when $G_{0}$ has constant dimension, using smooth bisections, which exist through each point of $G$. But we will need to apply the result to the case where $G_{0}$ has components of different dimensions, and so we take another approach.

Note that the action of $G$ on $Q_{A}$ can be encoded as an isomorphism $j: l^{!} Q_{A} \rightarrow$ $r^{!} Q_{A}$ of line bundles over $G$. Following Mackenzie [13] we use ! rather than * to denote pull-backs.) A section $\lambda$ of $Q_{A}$ is then $G$-invariant if and only if the pulled back sections satisfy $j l^{\prime} \lambda=r^{!} \lambda$. Moreover, since $l$ and $r$ are submersions, $\lambda$ is smooth in a neighborhood of $x \in G_{0}$ if and only if the pull-back $l^{!} \lambda$ (or $r ! \lambda$ ) is smooth in a neighborhood of some point of $l^{-1}(x)$ (or $r^{-1}(x)$ ). It follows easily that the set of points $x \in G_{0}$ near which an invariant section $\lambda$ is smooth is $G$-invariant, and the lemma follows.

We thus have:

COROLLARY 4.2. Let $G \Longrightarrow G_{0}$ be a groupoid, $G_{0}^{\prime}$ and $G_{0}^{\prime \prime}$ full open subsets. Then the restrictions from $G_{0}$ induce a natural isomorphism between smooth invariant sections of $Q_{A^{\prime}}$ and those of $Q_{A^{\prime \prime}}$.

To establish invariance of $Q_{A}$ under general Morita equivalences, we will use the following standard ${ }^{5}$ notion of "linking groupoids".

${ }^{5}$ It is hard to pinpoint the first occurrence of this notion, but it is implicit in work of Kumjian and Renault giving geometric constructions of linking algebras in the algebraic theory of Morita equivalence. The first explicit appearance of the term seems to be in [14]. 
PROPOSITION 4.3. Let $G^{\prime}$ and $G^{\prime \prime}$ be groupoids and $B$ an invertible $\left(G^{\prime}, G^{\prime \prime}\right)$-bibundle. Then there is a unique (up to natural isomorphism) groupoid $G$ such that:

1. $G_{0}$ is the disjoint union of $G_{0}^{\prime}$ and $G_{0}^{\prime \prime}$.

2. $G^{\prime}$ and $G^{\prime \prime}$ are the restrictions of $G$ to $G_{0}^{\prime}$ and $G_{0}^{\prime \prime}$, respectively.

3. The set $l^{-1}\left(G_{0}^{\prime}\right) \cap r^{-1}\left(G_{0}^{\prime \prime}\right)$ is identified with $B$ in such a way that the projections of $B$ to $G_{0}^{\prime}$ and $G_{0}^{\prime \prime}$ and the left and right actions on the bibundle $B$ with left and right multiplication in the groupoid $G$.

Proof. We assume without loss of generality that $B$ is disjoint from $G^{\prime}$ and $G^{\prime \prime}$. Let $G$ be the disjoint union $G^{\prime} \cup B \cup \bar{B} \cup G^{\prime \prime}$, where $\bar{B}$ is a copy of $B$. The inversion operation on $G$ is defined to be the union of those on $G^{\prime}$ and $G^{\prime \prime}$ and the correspondence between $B$ and $\bar{B}$, along with its inverse. The groupoid structure on $G$ is then completely determined by the conditions enumerated in the statement of the Proposition.

Combining Corollary 4.2 and Proposition 4.3, we obtain the main result of this section. The proof of the last part of the following statement is left to the reader.

THEOREM 4.4. Let $G^{\prime}$ and $G^{\prime \prime}$ be groupoids and $B$ an invertible $\left(G^{\prime}, G^{\prime \prime}\right)$-bibundle. Then $B$ induces an isomorphism between the $G$-invariant sections of $Q_{A^{\prime}}$ and the $G^{\prime}$-invariant sections of $Q_{A^{\prime \prime}}$. Isomorphic bibundles induce the same isomorphism, and the composition of bibundles induces the composed isomorphism.

Remark 4.5. A more formal way of stating the results above is that there is a 2 -functor from the 2-category of groupoids, invertible bibundles, and isomorphisms of invertible bibundles to the "discrete" 2-category of vector spaces, isomorphisms of vector spaces, and trivial isomorphisms of isomorphisms. This is very reminiscent of the decategorification construction of [1], except that it is not clear how it extends to more general morphisms. It may be that the more appropriate construction is that which produces (sometimes partially defined) morphisms of vector spaces of distributions from suitable canonical relations between cotangent bundles, as suggested in the introduction.

\section{Transformation Groupoids}

Let $h \mapsto h_{X}$ be an action of a Lie group $H$ on a manifold $X$, and let $v \mapsto v_{X}$ be the corresponding Lie algebra action, which takes $v \in \mathfrak{h}$ to the vector field on $X$ which generates the 1-parameter group $(\exp (-t v))_{H}$ of diffeomorphisms. (We need the minus sign to get a representation rather than an antirepresentation.) Let $G=$ $H \times X \Longrightarrow X=G_{0}$ be the corresponding transformation Lie groupoid. Its Lie algebroid is the trivial bundle $A=\mathfrak{h} \times X$, and the anchor is $(v, x) \mapsto v_{X}(x)$. Any section of $Q_{A}$ may be factored as $a^{-1} b$, where $a$ is a constant element of $\wedge^{\text {top }} \mathfrak{h}^{*}$ and $b$ is a form of top degree on $X$. If $H$ is unimodular, we can take $a$ to be adjointinvariant, in which case $G$-invariance of $a^{-1} b$ is equivalent to $H$-invariance of $b$. 
If $H$ is not unimodular, then, for $a^{-1} b$ to be invariant, we must have, for each $h \in$ $H, h_{X}^{*} b=\mu(h) b$, where $\mu: H \rightarrow \mathbb{R}^{\times}$is the modular function of $H$ (i.e. the determinant of the adjoint representation).

Whether or not the group $H$ is unimodular, the transformation groupoid is unimodular as long as the action (and hence the groupoid) is proper, which we will assume from now on. As a result, we can choose $a$ and $b$ as required above. Since the isotropy groups are compact, the modular function is identically 1 on them, and so the form $b$ will be $H$-invariant.

If $H$ is compact, we may apply Definition 2.3 to any relatively compact region in $X / H$ to conclude that the induced measure there, pushed forward from $X / / H$, is $\left(\int_{H} a_{r}\right)^{-1} b$. In particular, if the integral of $b$ over $X$ is finite, we have

$$
\operatorname{vol}_{(a, b)}(X / / H)=\operatorname{vol}_{b}(X) / \operatorname{vol}_{a}(H),
$$

exactly as in the finite case.

If $H$ is not compact, we must use Definition 3.3. As in the discussion leading up to that definition, we choose, over the regular part of $G_{0}$, an invariant section $\alpha$ of $\wedge^{\text {top }} \operatorname{ker} \rho^{*}$, i.e. an invariant family of bi-invariant measures on the isotropy groups. These, together with $a$, induce a section of $\bigwedge^{\text {top }}(A / \operatorname{ker} \rho)^{*}$. This section is transferred by $\rho$ itself to a section of $\Lambda^{\text {top }} \rho(A)^{*}$, which with $b$ then induces an invariant section $\beta$ of $\operatorname{coker} \rho$, i.e. a top-degree form on the orbit space. We may then use the formula in Definition 3.3 to compute the measure on $X / / H$ arising from the invariant section $a^{-1} b$ of $Q_{A}$.

EXAMPLE 5.1. Let $X$ be the Euclidean plane with its usual Euclidean measure $b=\mathrm{d} x \wedge \mathrm{d} y=r \mathrm{~d} r \wedge \mathrm{d} \theta$, and $H$ the group $S O(2)$ acting in the usual way by rotations around the origin. Let $a$ be the usual angle measure on $S O(2)$. Then the isotropy groups of regular points are trivial; if we take counting measure on them, the induced measure on the regular orbit space $\mathbb{R}^{+}$with coordinate $r$ is the quotient $(\mathrm{d} \theta)^{-1} \otimes r \mathrm{~d} r \wedge \mathrm{d} \theta$, which may be identified with $r \mathrm{~d} r$. The measure on the stack $\mathbb{R}^{2} / / S O(2)$ is then given by $r \mathrm{~d} r$.

Suppose, now, that we replace $S O(2)$ by the full orthogonal group $O(2)$. Since the adjoint representation of $O(2)$ is no longer orientation preserving, we must work with densities rather than forms, but we may take the infinitesimal data $a^{-1} b$ to be essentially the same as before. The main difference here is that the isotropy groups are now $\mathbb{Z}_{2}$, so that the measure on the stack $\mathbb{R}^{2} / / O(2)$ becomes $\frac{1}{2} r \mathrm{~d} r$.

\subsection{STACKS OF CONJUGACY CLASSES AND ADJOINT ORBITS}

Let $H$ be any Lie group acting on either $H$ itself by conjugation or on the Lie algebra $\mathfrak{h}$ by the adjoint representation. In either case, the space $X$ on which $H$ is acting carries a natural isomorphism from $\bigwedge^{\text {top }} T^{*} X$ to the trivial bundle with fibre $\wedge^{\text {top }} \mathfrak{h}^{*}$. As a result, the bundle $Q_{A}$ has a natural invariant trivialization, so the groupoid is unimodular, and we may use the constant section 1 (i.e. choosing 
$a$ and $b$ to be "the same") to compute "canonical" measures on $H / / H$ and $\mathfrak{h} / / H$ when the action is proper. In fact the action is proper just when $H$ is compact. In this case we have $\operatorname{vol}_{1}(H / / H)=1$, while the volume of $\mathfrak{h} / / H$ is infinite. In either case, it is still interesting to compute the induced measure. We concentrate on the case of the action on $\mathfrak{h}$, since that on $H$ is related to it by the exponential map, which is equivariant. (The regular part of $H$ is contained in the set of regular values of the exponential map.)

To compute the induced measure, we begin by choosing invariant measures on the isotropy groups of the strongly regular elements, i.e. on the maximal tori. For convenience, we choose the measures for which the total volume is 1 .

Following [9], we choose a basis of the Lie algebra $\mathfrak{h}$ of the form

$$
\left(\mathbf{e}_{1}, \ldots, \mathbf{e}_{r}, \mathbf{f}_{1}, \ldots, \mathbf{f}_{k}, \mathbf{g}_{1}, \ldots, \mathbf{g}_{k}\right),
$$

where the first $r$ entries are a basis of a Cartan subalgebra $t$ which are also a basis for the lattice $\exp ^{-1}(e) \cap \mathfrak{t}$, and, for each $j, \mathbf{f}_{j}$ and $\mathbf{g}_{j}$ span a plane on which the adjoint action of $\mathfrak{t}$ is given by $\left[\mathbf{e}, \mathbf{f}_{j}\right]=\sigma_{j}(\mathbf{e}) \mathbf{g}_{j}$ and $\left[\mathbf{e}, \mathbf{g}_{j}\right]=-\sigma_{j}(\mathbf{e}) \mathbf{f}_{j}$ for linear forms $\sigma_{1}, \ldots, \sigma_{k}$ in $\mathfrak{t}^{*}$. The dual basis will be denoted $\left(\mathbf{e}_{1}^{*}, \ldots, \mathbf{e}_{r}^{*}, \mathbf{f}_{1}^{*}, \ldots, \mathbf{f}_{k}^{*}\right.$, $\left.\mathbf{g}_{1}^{*}, \ldots, \mathbf{g}_{k}^{*}\right)$, As measure ${ }^{6}$ on $\mathfrak{t}$ we choose $\mathbf{e}_{1}^{*} \wedge \cdots \wedge \mathbf{e}_{r}^{*}$, which has total volume 1 on $T$, and on $\mathfrak{h}$ we take

$$
\mathbf{e}_{1}^{*} \wedge \cdots \wedge \mathbf{e}_{r}^{*} \wedge \mathbf{f}_{1}^{*} \wedge \cdots \wedge \mathbf{f}_{k}^{*} \wedge \mathbf{g}_{1}^{*} \wedge \cdots \wedge \mathbf{g}_{k}^{*} .
$$

This induces the measure $\mathbf{f}_{1}^{*} \wedge \cdots \wedge \mathbf{f}_{k}^{*} \wedge \mathbf{g}_{1}^{*} \wedge \cdots \wedge \mathbf{g}_{k}^{*}$ on $\mathfrak{h} / \mathfrak{k}=\mathfrak{h} / \operatorname{ker} \rho$.

We now use the anchor map $\rho$ (or, more precisely, $\rho^{-1^{*}}$ ), to transfer the last measure to the tangent space $\rho(\mathfrak{h})$ to the adjoint orbit through a typical point $\mathbf{e}$ (which may be taken in $t$ since the latter intersects every orbit). Since, at the basepoint $\mathbf{e} \in \mathfrak{t}, \rho$ is just $[\mathbf{e}, \cdot]$, so that $\rho^{-1^{*}}\left(\mathbf{f}_{j} \wedge \mathbf{g}_{j}\right)=\left(\sigma_{j}(\mathbf{e})\right)^{-2} \mathbf{f}_{j} \wedge \mathbf{g}_{j}$, we obtain the measure

$$
\prod_{j}\left(\sigma_{j}(\mathbf{e})\right)^{-2} \mathbf{f}_{1}^{*} \wedge \cdots \wedge \mathbf{f}_{k}^{*} \wedge \mathbf{g}_{1}^{*} \wedge \cdots \wedge \mathbf{g}_{k}^{*}
$$

on the tangent space to the adjoint orbit. Dividing the given measure on $\mathfrak{h}$ by this one, we obtain

$$
\prod_{j}\left(\sigma_{j}(\mathbf{e})\right)^{2} \mathbf{e}_{1}^{*} \wedge \cdots \wedge \mathbf{e}_{r}^{*}
$$

on the normal space to the orbit, i.e. on the tangent space to $\mathfrak{h} / H$, identified with a tangent space to $t$.

\footnotetext{
${ }^{6}$ We will abuse language by using the term "measure" to refer to top degree forms, and sometimes even to particular values of these forms.
} 
Since the volume of each isotropy group in the chosen measure is equal to 1 , the expression (2) is also the induced measure on the stack $\mathfrak{h} / / \mathrm{H}$. We may compare this expression with a calculation in [9]. According to the formula there on the bottom of p. 22, for any function $\phi$ on $\mathfrak{h} / H$,

$$
\int_{\mathfrak{h}} \phi(p(X)) \mathrm{d} X=\int_{\mathfrak{t}^{+}} \prod_{j}\left(\sigma_{j}(\mathbf{e})\right)^{2} \phi(\mathbf{e}) \mathrm{d} \mathbf{e},
$$

where $\mathfrak{h} / H$ is identified with the positive Weyl chamber $\mathfrak{t}^{+}, p: \mathfrak{h} \rightarrow \mathfrak{h} / H$ is the natural projection, $\mathrm{d} X$ is the measure on $\mathfrak{h}$ which agrees at 0 (via the exponential map) with the invariant measure on $H$ with total volume 1 , and $\mathrm{d} \mathbf{e}=\mathbf{e}_{1}^{*} \wedge \cdots \wedge \mathbf{e}_{r}^{*}$. Thus, our "natural" measure on $\mathfrak{h} / / H$, which involved no choices, may be identified with the push-forward under $p$ of the measure $\mathrm{d} X$ on $\mathfrak{h}$ normalized as described above.

If the group $H$ is noncompact, but is semisimple and reductive, its adjoint representation still defines a proper action when restricted to the open subset $\mathcal{D}_{H} \subset \mathfrak{h}$ of strongly stable elements. These are defined in [18], where it is shown that the adjoint action of $H$ on $\mathcal{D}_{H}$ is proper. Several characterizations of strongly stable elements are given there; the shortest of these to state is that the strongly stable elements are those which belong to the Lie algebra of a unique maximal compact subgroup of $K$. It is also shown there (in slightly different terms) that the action groupoid $H \times \mathcal{D}_{H} \Longrightarrow \mathcal{D}_{H}$ is equivalent to its restriction $K \times \mathcal{E}_{K} \Longrightarrow \mathcal{E}_{K}$, where $K$ is any maximal compact subgroup of $H$ and $\mathcal{E}_{K}=\mathcal{D}_{H} \cap \mathfrak{k}$. (The elements $v$ of $\mathcal{E}_{K}$ are characterized by the condition that the vector field $\mu_{S}$ on the symmetric space $S=H / K$ given by the infinitesimal action of $\mu \in \mathcal{H}$ has a nondegenerate zero at the coset $e K$.) Thus, the natural measure on $\mathcal{D}_{H} / / H$ is the same as that on $\mathcal{E}_{K} / / K$.

A similar reduction is possible for the conjugation action of $H$ on itself, following the analysis of Demazure [8].

\subsection{SYMPLECTIC GROUPOIDS AND POISSON MANIFOLDS}

A Lie groupoid $G \Longrightarrow G_{0}$ is a symplectic groupoid if $G$ is equipped with a symplectic structure $\omega$ which is multiplicative in the sense that $m^{*} \omega=\omega_{1}+\omega_{2}$ on the space $G_{2} \subset G \times G$ of composable pairs, where $m: G_{2} \rightarrow G$ is the product operation, and $\omega_{1}$ and $\omega_{2}$ are the pull-backs of $\omega$ by the first and second projections from $G_{2}$ to $G$. Some standard facts (see, for example [17]) about symplectic groupoids are: $G_{0}$ carries a unique Poisson structure $\Pi$ for which $l$ and $r$ are Poisson and anti-Poisson maps, respectively; the units form a lagrangian submanifold of $G$; the Lie algebroid of $G$ is naturally isomorphic to $T^{*} G_{0}$ with the anchor map $T^{*} G_{0} \rightarrow T G_{0}$ given by $\Pi$ and a bracket for which $\{\mathrm{d} f, \mathrm{~d} g\}=\mathrm{d}\{f, g\}$ for all functions $f$ and $g$ on $G_{0}$. Conversely, if $G_{0}$ is any Poisson manifold for which the associated Lie algebroid structure on $T^{*} G_{0}$ is integrable to a groupoid, then the integrating groupoid with simply-connected source fibres is a symplectic groupoid 
with underlying Poisson manifold $G_{0}$. If we allow integration by stacks rather than just manifolds, then there is a bijective (up to natural isomorphisms) correspondence between Poisson manifolds and symplectic groupoids with simply-connected source fibres [15].

Writing $A$ as usual for the Lie algebroid $T^{*} G_{0}$, we have a natural isomorphism between $Q_{A}$ and the tensor square $\left(\bigwedge^{\text {top }} T^{*} G_{0}\right)^{\otimes 2}$. $Q_{A}$ therefore carries a natural orientation, and its positive sections are the squares of nowhere-vanishing sections $v$ of $\bigwedge^{\text {top }} T^{*} G_{0}$. Assuming $G$ to have connected source fibres, we have (whether or not $v$ is nowhere-vanishing) that $v^{2}$ is $G$-invariant exactly when $v$ is invariant under all hamiltonian vector fields.

\subsection{SYMPLECTIC MANIFOLDS}

Let $\left(S, \omega_{S}\right)$ be a $2 m$-dimensional, connected ${ }^{7}$ symplectic manifold, considered as a Poisson manifold. The source-connected symplectic groupoids for $S$ (all of them transitive) are just the quotients of the fundamental groupoid $\pi(S)$ associated with normal subgroups of the fundamental group of $S$. For any such groupoid, the invariant sections of $Q_{A}$ are just the constant multiples $\lambda=c v_{S}^{2}$ of the square of the Liouville measure $\nu_{S}=\frac{(-1)^{m(m-1) / 2}}{m !} \omega_{S}^{m}$.

Now let $G \Longrightarrow G_{0}$ be the symplectic groupoid of $S$ associated with the subgroup $K$ of the fundamental group. This groupoid is proper when $K$ is finite; two natural choices are the trivial group and the fundamental group itself, when it is finite. It is equivalent to the groupoid $K \Longrightarrow \mathrm{pt}$; i.e. $G_{0} / G$ is just a point, and $G_{0} / / G=B K$. We recall from the Introduction that $\#(B K)=1 / \#(K)$ (in particular, we get the value 1 when $K$ is the trivial group and $G$ is the pair groupoid), but we are interested here in the volume $\operatorname{vol}(B K)$, which will depend on the choice of the constant $c$ in $\lambda$. To compute this volume, we begin by factoring $c v_{s}^{2}$ as the product of the sections $a^{-1}=c v_{s}$ of $\bigwedge^{\text {top }} A^{*}$ and $b=v_{S}$ of $\bigwedge^{\text {top }} T^{*} G_{0}$. Next, we choose the section 1 of the trivial bundle $\operatorname{ker} \rho$. Noting first that integrating 1 over the isotropy groups will give \#(K), we next observe that the induced section of $\Lambda^{\text {top }}(A / \operatorname{ker} \rho)^{*}$ is again $a^{-1}=c v_{S}$. Its inverse is the section $a=c^{-1} v_{S}^{-1}$ of $\bigwedge^{\text {top }}(A / \operatorname{ker} \rho)=\bigwedge^{\text {top }}\left(T^{*} G_{0}\right)^{*}$. Now we must transfer this to a section of $\bigwedge^{\text {top }} T^{*} G_{0}$ by using $\rho^{-1^{*}}$, with $\rho: T^{*} S \rightarrow T S$ given by the Poisson structure inverse to $\omega_{S}$. It is not hard to see (for instance by using symplectic bases) that $\rho^{*}$ pulls back $v_{S}$ to $v_{S}^{-1}$, so $\rho^{-1^{*}}$ transfers $c^{-1} v_{S}^{-1}$ to $c^{-1} v_{S}$. Finally, we divide this measure along the "orbits" into the measure $v_{S}$ on the base of the groupoid to obtain the measure $c$ on the orbit space. Since this orbit space consists of a single point, we conclude that $\operatorname{vol}_{c v_{S}^{2}}\left(G_{0} / / G\right)=c / \#(K)$. Observing that this agrees with the cardinality just when $c=1$, we are led to the conclusion that the Liouville measure

${ }^{7}$ From the point of view of Poisson geometry, we should perhaps call a manifold with nondegenerate closed 2-form "symplectic" only if it is connected, since otherwise it has more than one symplectic leaf! 
(including the factor of $1 / n$ !) is really the natural one on a symplectic manifold. (Of course, this is just a consequence of the fact that the Liouville measure is the only one, up to sign, which corresponds to its inverse by the Poisson structure.)

\subsection{REGULAR POISSON MANIFOLDS}

We look here at a very simple example. Let $G_{0}=P=\Sigma \times \mathbb{R}$ be the Poisson manifold given by a family $\omega_{t}$ of symplectic structures on a 2 -sphere $\Sigma$, parametrized by $t \in \mathbb{R}$. If the area $V(t)=\int_{\Sigma} \omega_{t}$ of the symplectic leaf $\Sigma \times\{t\}$ has no critical points as a function of $t$, then this Poisson manifold is integrable. (See, for example, Sections 5 and 7 of [7]). Its symplectic groupoid $G$ is a circle bundle over $\Sigma \times \Sigma \times \mathbb{R}$ for which the map $(l, r)$ takes the entire fibre over $(x, y, t)$ to $((x, t),(y, t))$. The fibres over the diagonal points $(x, t),(x, t)$ are the isotropy groups, and the corresponding Lie algebra bundle is naturally isomorphic to $T^{*} \mathbb{R}$. (For any Poisson manifold at a regular point, the isotropy algebras of a symplectic groupoid are naturally isomorphic to the conormal spaces of the symplectic leaves.) Under this isomorphism, the kernel of the exponential map from the isotropy algebra at $(x, t)$ to the corresponding isotropy group consists of the integer multiples of $\mathrm{d} V=V^{\prime}(t) \mathrm{d} t$.

Let us now choose the section $\lambda=f(t)\left(\omega_{t} \wedge \mathrm{d} t\right)^{2}$ of $Q_{A}$ and compute the corresponding measure on the stack $G_{0} / / G$, which is a bundle of $B S^{1}$ 's over $\mathbb{R}$. As we did in the symplectic case, we factor $\lambda$ as the product of the sections $a^{-1}=$ $f(t) \omega_{t} \wedge \mathrm{d} t$ of $\wedge^{\text {top }} A^{*}$ and $b=\omega_{t} \wedge \mathrm{d} t$ of $\wedge^{\text {top }} T^{*} G_{0}$. Writing $\tau$ for the coordinate on the isotropy groups corresponding to $\mathrm{d} t$, we choose the measure $\mathrm{d} \tau$, for which the volume of the isotropy group at $(x, t)$ is equal to $V^{\prime}(t)$. The induced measure on $A / \operatorname{ker} \rho$ is then $f(t)^{-1}\left(\omega_{t}\right)^{-1}$, which pushes forward under $\rho^{-1^{*}}$ to the measure $f(t)^{-1} \omega_{t}$ along the symplectic leaves. Dividing this into the measure $\omega_{t} \wedge \mathrm{d} t$ on the base of our groupoid gives the measure $f(t) \mathrm{d} t$ on the quotient space. Using now the fact that the volume of the isotropy group at $t$ is $V^{\prime}(t)$, we find that the measure on the stack $G_{0} / / G$ is equal to $\left(f(t) / V^{\prime}(t)\right) \mathrm{d} t$. To better understand this result, we assume $f$ positive, so that $\lambda$ is the square of the measure $(f(t))^{1 / 2} \omega_{t} \wedge \mathrm{d} t$ on $P$. Dividing the latter by the Liouville measure along the leaves gives $(f(t))^{1 / 2} \mathrm{~d} t$ as "quotient" measure on $\mathbb{R}$. It agrees with the stack measure just when $\lambda$ is the square of $\omega_{t} \wedge V^{\prime}(t) \mathrm{d} t=\omega_{t} \wedge \mathrm{d} V(t)$.

We may interpret the last calculations as meaning that, just as Liouville measure is a natural measure on a symplectic manifold, so $\mathrm{d} V$ is a "natural" measure on the leaf space of the Poisson manifold above. A possible generalization to arbitrary regular Poisson manifolds goes as follows.

Let $G_{0}=P$ be a Poisson manifold which is strongly regular in the sense that its symplectic leaves are the fibres of a smooth fibration $P \rightarrow M$. Following [7], we introduce the lattice ("réseau") $\Lambda \subset T^{*} M$ of differentials of periods of the symplectic forms on the leaves. It is a (not necessarily closed) lagrangian submanifold of $T^{*} M$ for which the projection to $M$ is a local diffeomorphism and whose 
intersection with each fibre is a (discrete) additive subgroup. The Poisson manifold is integrable to a symplectic groupoid when $\Lambda$ is closed and a covering space of $M$.

We will assume further, for simplicity, that the leaves are simply connected and that the intersection of $\Lambda$ with each cotangent space is a full lattice, in which case the quotient $T^{*} M / \Lambda$ is a bundle of tori whose pull-back to $P$ is the isotropy subgroupoid of the symplectic groupoid $G$ of $P$. (The assumption that the leaves are simply connected implies that the symplectic groupoid is unique up to isomorphism.) In this case, the lattice defines an integrable $G L(n, \mathbb{Z})$ structure on $M$ which determines (up to sign) a natural measure.

CONJECTURE 5.2. With notation as above, if the section $\lambda$ of $Q_{A}$ is the square of an invariant measure on $P$, factored as the product of the Liouville measure along the symplectic leaves and (the pull-back of) a measure $\beta$ on the leaf space $M$, then the induced measure on the stack $P / / G$ agrees with $\beta$ if and only $\beta$ is the measure associated to the integer affine structure on $M$.

\subsection{DUALS OF LIE ALGEBRAS}

If $H$ is a compact Lie group, its coadjoint representation on $\mathfrak{h}^{*}$ is equivalent, via a bi-invariant metric, to its adjoint representation on $\mathfrak{h}$. Different choices of the metric will lead to different identifications, hence the canonical section of $Q_{A}$ for the adjoint action does not lead to a canonical section of $Q_{A}$ for the coadjoint action.

In fact, the natural structure on $\mathfrak{h}^{*}$ is its Lie-Poisson structure, for which $T^{*} G$ is a symplectic groupoid. On an open dense subset, this Poisson structure is regular and satisfies the hypotheses of the conjecture at the end of the previous section. For the special case of $S U(2)$, the Poisson structure on the complement of the origin has concentric spheres as its symplectic leaves, and we may choose a linear radial coordinate $t$ so that the symplectic area of the sphere of radius $t$ is $V(t)=4 \pi t$. The "natural" measure on the leaf space is then $4 \pi \mathrm{d} t$, whose product with the Liouville measure along the leaves is $\omega_{t} \wedge 4 \pi \mathrm{d} t=4 \pi t \omega_{1} \wedge \mathrm{d} t$. Notice that this is not a translation-invariant measure!

\section{Acknowledgements}

I would like to think John Baez, Kai Behrend, Rui Loja Fernandes, Minhyong Kim, Yvette Kosmann-Schwarzbach, Eckhard Meinrenken, Martin Olsson for helpful discussion and comments. I would also like to thank the group Analyse Algébrique at the Institut Mathématique de Jussieu for their hospitality.

Open Access This article is distributed under the terms of the Creative Commons Attribution Noncommercial License which permits any noncommercial use, distribution, and reproduction in any medium, provided the original author(s) and source are credited. 


\section{References}

1. Baez, J., Dolan, J.: From Finite Sets to Feynman Diagrams, Mathematics Unlimited2001 and Beyond, pp. 29-50. Springer, Berlin (2001)

2. Baez, J., Hoffnung, A.E., Walker, C.D.: Groupoidification Made Easy. arXiv preprint 0812.4864[math.QA]

3. Behrend, K.A.: The Lefschetz trace formula for algebraic stacks. Invent. Math. 112, 127-149 (1993)

4. Behrend, K.: Cohomology of Stacks, Intersection Theory and Moduli. ICTP Lecture Notes, vol. XIX, pp. 249-294 (electronic). Abdus Salam Int. Cent. Theoret. Phys., Trieste (2004)

5. Behrend, K., Xu, P.: Differentiable Stacks and Gerbes. arXiv preprint math.DG/ 0605694

6. Crainic, M.: Differentiable and algebroid cohomology, van Est isomorphisms, and characteristic classes. Comment. Math. Helv. 78, 681-721 (2003)

7. Dazord, P.: Groupoïdes symplectiques et troisième théorème de Lie "non linéaire". Lect. Notes Math. 1416, 39-74 (1990)

8. Demazure, M.: Sur le théorème de Krein-Gel'fand-Lidskii. C. R. Acad. Sci. Paris 286, 625-626 (1978)

9. Dooley, A.H., Wildberger, N.J.: Harmonic analysis and the global exponential map for compact Lie groups. Funct. Anal. Appl. 27, 21-27 (1993)

10. Evens, S., Lu, J.-H., Weinstein, A.: Transverse measures, the modular class, and a cohomology pairing for Lie algebroids. Q. J. Math 50, 417-436 (1999)

11. Kim, M.: Lefschetz trace formula for equivariant cohomology. Ann. Sci. de l'É.N.S. 28, 669-688 (1995)

12. Kosmann-Schwarzbach, Y., Laurent-Gengoux, C., Weinstein, A.: Modular classes of Lie algebroid morphisms. Transform. Groups 13, 727-755 (2008)

13. Mackenzie, K.C.H.: General Theory of Lie Groupoids and Lie Algebroids. London Mathematical Society Lecture Note Series, vol. 213. Cambridge University Press, Cambridge (2005)

14. Muhly, P.: Bundles over groupoids. In: Ramsay, A., Renault, J. (eds.) Groupoids in Analysis, Geometry, and Physics. Contemp. Math. 282, 67-82 (2001)

15. Tseng, H.H., Zhu, C.: Integrating Poisson manifolds via stacks. Travaux Mathématiques 16, 285-297 (2005)

16. Tu, J.-L.: La conjecture de Novikov pour les feuilletages hyperboliques. $K$-Theory 16, 129-184 (1999)

17. Vaisman, I.: Lectures on the Geometry of Poisson Manifolds. Birkhäuser, Basel (1994)

18. Weinstein, A.: Poisson geometry of discrete series orbits, and momentum convexity for noncompact group actions. Lett. Math. Phys. 56, 17-30 (2001)

19. Weinstein, A.: Linearization of regular proper groupoids. Journal de l'Institut de Mathématiques de Jussieu 1, 493-511 (2002)

20. Weinstein, A.: The Maslov gerbe. Lett. Math. Phys. 69, 3-9 (2004)

21. Zung, N.T.: Proper groupoids and momentum maps: linearization, affinity, and convexity. Ann. Sci. de l'É.N.S. 39, 841-869 (2006) 